Pacific Journal of Mathematics

ON THE YON NEWMAN REGULARITY OF RINGS WITH 


\title{
ON THE VON NEUMANN REGULARITY OF RINGS WITH REGULAR PRIME FACTOR RINGS
}

\author{
Joe W. Fisher AND Robert L. SNider
}

\begin{abstract}
Kaplansky made the following conjecture: $\mathbf{A}$ ring $R$ is von Neumann regular if and only if $R$ is semiprime and each prime factor ring of $R$ is von Neumann regular. That the conjecture failed in general was shown by a counterexample of Snider. It is established that a necessary and sufficient condition for Kaplansky' conjecture to hold is that the union of every chain of semiprime ideals of $R$ be semiprime. From this theorem flow many corollaries including proofs of Kaplansky's conjecture in the known special cases of commutative rings and rings without nilpotent elements. Moreover, it is used repeatedly in previously unaccessable situations. In one such situation semiprime Azumaya algebras with prime ideals maximal are characterized as von Neumann regular algebras. In another a theorem of Armendariz and Fisher is proved which characterizes von Neumann regular polynomial identity rings (P. I.-rings) as fully idempotent rings.
\end{abstract}

With an eye to the commutative theory, one might be tempted to venture that Kaplansky's conjecture does hold for P. I.-rings. This is not the case. However, we prove in $\S 2$ that Kaplansky's conjecture does hold for stable P. I.-rings. Furthermore, if $R$ is a semiprime P. I.-ring with regular prime factor rings, we are able to prove that $R$ contains a large (essential) regular ideal.

Also in $\S 2$ we prove that if each prime factor ring of a ring $R$ is left $\pi$-regular, then $R$ is left $\pi$-regular. By using this we are able to characterize P. I.-rings with prime ideals maximal as $\pi$ regular rings. We conclude by proving that a von Neumann regular P. I.-ring contains a large (essential) biregular ideal.

Throughout this paper, $R$ will denote an associative ring which does not necessarily have a unity. A ring $R$ is called von Neumann regular or just regular if for each $a$ in $R$ there exists an $x$ in $R$ such that $a=a x a . \quad R$ is said to be $\pi$-regular if for every $a$ in $R$ there exists a positive integer $n$ (depending on $a$ ) and an element $x$ in $R$ such that $a^{n}=a^{n} x \alpha^{n} . R$ is called n-regular if for each $a$ in $R$ there exists an $x$ in $R$ such that $a^{n}=a^{n} x a^{n}$. Thus 1-regularity is von Neumann regularity. For basic properties of these classes of rings see [14]. We say that $R$ is a P. I.-ring if $R$ satisfies a polynomial identity with coefficients in the centroid and at least one coefficient is invertible. 
1. Kaplansky's conjecture. Kaplansky made the following conjecture in [18]: A ring $R$ is von Neumann regular if and only if $R$ is semiprime and each prime factor ring of $R$ is von Neumann regular. It is well-known that the conjecture holds for commutative rings [8, p. 173]. Snider [27] has shown that the conjecture fails to hold in general. Later, C. Lanski pointed out to us that the following example of Kaplansky [17] is also a counterexample.

EXAMPLE 1. Let $R$ consist of all sequences of 2 by 2 matrices over a field which are eventually strictly upper triangular. Then $R$ is semiprime and if $P$ is a prime ideal of $R$, then Posner's theorem [22] guarantees that $R / P$ has a simple Artinian classical quotient ring. By direct calculation it can be shown that nonzero divisors in $R / P$ are invertible in $R / P$. Thus $R / P$ coincides with its quotient ring and so $R / P$ is regular. Finally, $R$ is not regular since the constant sequence $a=\left(\left[\begin{array}{ll}0 & 1 \\ 0 & 0\end{array}\right]\right)$ is not a regular element.

Consider the condition: $(*)$ the union of every chain of semiprime ideals in $R$ is semiprime. We now show that condition $\left(^{*}\right)$ is precisely what is needed in order to make Kaplansky's conjecture valid and thereby produce necessary and sufficient conditions for a ring to be von Neumann regular.

THEOREM 1.1. A ring $R$ is von Neumann regular if and only if $R$ is semiprime, $R$ satisfies $\left({ }^{*}\right)$, and each prime factor ring of $R$ is von Neumann regular.

Proof. The proof in one direction is immediate. In the other direction, we need to show that for each $a$ in $R$ there exists an $r$ in $R$ such that $a=a r a$. Assume, to the contrary, that there exists an $a$ in $R$ such that $a=a x a$ has no solution in $R$. By using $\left(^{*}\right)$ and Zorn's lemma, we choose a semiprime ideal $A$ which is maximal with respect to the property that $\bar{a}=\overline{a x a}$ has no solution in $R / A$, i.e., $a-a x a$ is not in $A$ for every $x \in A$. Clearly $A$ is not prime. Hence by passing to $R / A$, we may assume that $A$ is zero and $R$ is semiprime but not prime. Therefore, there exist nonzero semiprime ideals $I$ and $J$ in $R$ such that $I \cap J=0$. By the choice of $A$, there exist $x$ and $y$ in $R$ with $a-a x a$ in $I$ and $a-a y a$ in $J$. Thus $a-a(x+y-x a y) a=(a-a x a)-a y a+(a x \alpha) y a$ and so $a-$ $a(x+y-x a y) a \equiv 0 \quad(\bmod I) . \quad$ Similarly $a-a(x+y-x a y) a \equiv 0$ $(\bmod J)$. Therefore, $a-a(x+y-x a y) a=0$. This contradicts the choice of $A$. Consequently $R$ is von Neumann regular and the proof is complete. 
REMARK 1. It is apparent that this technique of proof also yields: If $R$ is semiprime, $R$ satisfies $\left({ }^{*}\right)$, and each prime factor ring of $R$ is $n$-regular, then $R$ is $n$-regular.

One class of rings which satisfies condition $\left(^{*}\right)$ is the class of rings which Kaplansky [19] calls neocommutative, i.e., rings in which the product of two finitely generated ideals (finitely generated as two-sided ideals) is again finitely generated. In particular two-sided Noetherian rings, i.e., rings with the ascending chain condition on two-sided ideals are neocommutative. The prime radical of $R$, denoted $B(R)$, is the intersection of all prime ideals of $R$.

COROLlary 1.2. If $R$ is two-sided Noetherian and each prime factor ring of $R$ is von Neumann regular, then $R / B(R)$ is von Neumann regular and $B(R)$ is nilpotent.

Proof. Theorem 1.1 applied to $R / B(R)$ yields that it is regular. That $B(R)$ is nilpotent in this case is well-known.

Another class of rings which satisfies $(*)$ is the class in which each ideal is semiprime ( $f$-regular rings [7]), or equivalently, each ideal is idempotent (fully idempotent rings [10]). Included in this class is the class of biregular rings [14].

COROLLARY 1.3. A ring $R$ is von Neumann regular if and only if each factor ring of $R$ is semiprime and each prime factor ring of $R$ is von Neumann regular.

Proof. Evident.

If a ring $R$ has the property that each prime factor ring is a (not necessarily commutative) domain, then $R$ satisfies condition $\left(^{*}\right.$ ), since an ideal $I$ of $R$ is semiprime if and only if $r^{2}$ in $I$ implies $r$ in $I$. Rings which have the property that prime factor rings are domains include commutative rings as well as rings in which prime factor rings are division rings. Hence we immediately have the following corollary which was discovered independently by various authors including Herstein, Snider [27], Steinberg [28], and Wong [30]. A prime ideal $P$ of $R$ is completely prime if $R / P$ is a domain.

COROLlaRY 1.4. Let $R$ be a ring without nilpotent elements. Then $R$ is von Neumann regular if and only if each completely prime factor ring of $R$ is von Neumann regular.

Proof. One direction is clear. In the other direction each minimal prime ideal of $R$ is completely prime by Andrunakievic and 
Rjabuhin [2]. Therefore, for each proper ideal $P$ of $R, R / P$ is a division ring. Wherefore $R$ is regular by Theorem 1.1.

A ring $R$ is called restricted von Neumann regular if for each nonzero ideal $I$ of $R$, the factor ring $R / I$ is von Neumann regular. See S. K. Jain and Saroj Jain [15].

COROLLARY 1.5. A restricted von Neumann regular ring which is semiprime but not prime is von Neumann regular.

Proof. Evident.

2. P. I.-rings with regular prime factor rings. A ring $R$ is left $\pi$-regular if for each $a$ in $R$ there exists an $x$ in $R$ and a positive integer $n$ such that $a^{n}=x a^{n+1}$. Note that left $\pi$-regularity of $R$ is equivalent to each descending chain of left ideals of the form $R a \supset R a^{2} \supset R a^{3} \supset \cdots$ terminating. Right $\pi$-regularity is defined analogously.

THEOREM 2.1. The following are equivalent for a ring $R$ :

(a) $R$ is left (right) $\pi$-regular.

(b) Each prime factor ring of $R$ is left (right) $\pi$-regular.

Proof. That (a) implies (b) is clear. Suppose that (b) holds. We need to show that for each $a \in R$ there exists a positive integer $n$ such that $R a^{n}=R a^{n+1}$. Assume to the contrary that there exists an $a \in R$ such that the descending chain $\left\{R a^{i}: i=1,2, \cdots\right\}$ of left ideals does not terminate. By using Zorn's lemma we choose an ideal $I$ of $R$ which is maximal with respect to the property that the descending chain $\left\{R a^{i}+I: i=1,2, \cdots\right\}$ does not terminate. Clearly $I$ is not prime. By passing to $R / I$, we may assume that $I=0$ and $R$ is not a prime ring. Hence there exist nonzero ideals $J$ and $K$ in $R$ such that $J K=0$. By the choice of $I$, there exists a postivie integer $m$ such that $a^{m} \equiv x a^{m+1} \equiv x^{r} a^{m+r}(\bmod J)$ and $a^{m} \equiv y a^{m+1} \equiv y^{r} a^{m+r}(\bmod K)$ for any $r$. Take $r=m+1$, and from $\left(a^{m}-x^{r} a^{m+r}\right)\left(a^{m}-y^{r} a^{m+r}\right)=0$, we deduce that $a^{2 m}=z a^{2 m+1}$ for some $z \in R$. This contradicts the choice of $I$. Therefore, $R$ is left $\pi$ regular and the proof is complete.

Example 1 shows that no analogue of this theorem exists if left (right) $\pi$-regularity is replaced by regularity, biregularity or $f$-regularity. Also, the following example of J. D. McKnight, Jr. shows that no analogue exists if left (right) $\pi$-regularity is replaced by $\pi$-regularity.

EXAMPLE 2. Let $V$ be a countably infinite dimensional vector 
space over a field $F$ and let $\left\{v_{1}, v_{2}, \cdots\right\}$ be a basis for $V$. Let $S$ be the ring of all linear transformations on $V$. Set $R=\left[\begin{array}{ll}S & S \\ 0 & 0\end{array}\right]$. If $P$ is a prime ideal of $R$, then $P \supseteqq\left[\begin{array}{ll}0 & S \\ 0 & 0\end{array}\right]=I$. Therefore, $R / P$ is von Neumann regular since $R / I$ is regular. In order to see that $R$ is not $\pi$-regular, define $a \in S$ by $a v_{i}=v_{i+1}$ for each $i$ and $b \in S$ by $b v_{1}=0, b v_{i}=v_{i-1}$ for $i>1$. It can easily be shown that $\left[\begin{array}{ll}a & b \\ 0 & 0\end{array}\right]$ is not a $\pi$-regular element.

A ring $R$ is said to be of bounded index if the indices of the nilpotent elements in $R$ are bounded.

COROLlary 2.2. If $R$ is of bounded index, then the following are equivalent:

(a) $R$ is $\pi$-regular.

(b) Each prime factor ring of $R$ is left (right) $\pi$-regular.

Proof. Azumaya [6, Theorem 5] proves that the concepts of $\pi$-regularity and left (right) $\pi$-regularity coincide for rings of bounded index.

Another corollary is the following theorem. For a proof of the commutative analogue, see Storrer [29, Lemma 5.6].

THeOREM 2.3. Let $R$ be a P. I.-ring. Then the following are equivalent:

(a) $R$ is $\pi$-regular.

(b) Each prime ideal of $R$ is primitive.

(c) Each prime ideal of $R$ is maximal.

(d) $R$ is left (right) $\pi$-regular.

(e) $R / B(R)$ is $\pi$-regular.

(f) Each prime factor ring of $R$ is von Neumann regular.

Proof. If (a) holds and $P$ is a prime ideal of $R$, then Posner's theorem [22] yields that $R / P$ has a primitive Artinian classical twosided quotient ring which is forced to coincide with $R / P$ by the $\pi$ regularity of $R / P$. Hence (a) implies (b). That (b) implies (c) follows from Kaplansky's theorem [16]. Assume that (c) holds. By the strengthened version of Posner's theorem [25] prime factor rings of $R$ are left (right) $\pi$-regular. Whence $R$ is left (right) $\pi$-regular by Theorem 2.1 and (c) implies (d). Suppose that (d) holds. Then $R$ left (right) $\pi$-regular implies that $R / B(R)$ is left (right) $\pi$-regular. Since $R / B(R)$ is a semiprime P. I.-ring, it is of bounded index by Levitzki [20, Theorem 1]. Hence $R / B(R)$ is $\pi$-regular again in Azumaya [6, Theorem 5] and so (d) implies (e). The proof that (e) implies (f) is the same as the proof of (a) implies (b). Finally, 
assume that (f) holds. Evidently (f) implies (c). Then, since we have established (c) implies (d), $R$ is both left and right $\pi$-regular. Accordingly, for each $a$ in $R$ there exists a positive integer $n$ such that simulaneously $R a^{n}=R a^{2 n}$ and $a^{n} R=a^{2 n} R$, i.e., there exists an $x$ and $y$ in $R$ such that $a^{n}=x a^{2 n}$ and $a^{n}=a^{2 n} y$, in fine, $a^{2 n}=a^{2 n} y x a^{2 n}$ and $R$ is $\pi$-regular.

REMARK 2. In [17, p. 74] Kaplansky asks what can be deduced about a ring that is left $\pi$-regular and does left $\pi$-regularity enable one to construct idempotents? That (d) implies (a) in Theorem 2.3 establishes that the answer is affirmative for P. I.-rings by showing that left $\pi$-regular P. I.-rings are $\pi$-regular. Consequently each nonnil left ideal contains a nonzero idempotent which can easily be constructed.

COROLlaRY 2.4. If $R$ is a semiprime ring which satisfies a polynomial identity of degree $2 n$ and if each prime factor ring of $R$ is von Neumann regular, then $R$ is n-regular.

Proof. As before each prime factor ring of $R$ is simple Artinian. By Kaplansky's theorem [16] the dimension of each prime factor ring over its center is not greater than $n^{2}$. It follows that $R$ is of bounded index $n$. An application of Theorem 2.3 yields that $R$ is $\pi$-regular. Consequently $R$ is $n$-regular by Azumaya [6, Theorem 4].

REMARK 3. Since commutative rings satisfy a polynomial identity of degree 2, we obtain in this special case the commutative theorem which inspired Kaplansky's conjecture.

Example 1 shows that Kaplansky's conjecture is not valid for P. I.-rings. However, in that example, the ideal which consists of all sequences that are eventually zero is a von Neumann regular ideal which is essential, i.e., it has nonzero intersection with each nonzero ideal. We proceed to prove that this is entirely typical. We say that a P. I.-ring is stable of degree $n$ if it satisfies a polynomial identity of degree $n$ and no nonzero factor ring of $R$ satisfies a polynomial identity of degree less than $n$. See Small [26].

Theorem 2.5. Let $R$ be a semiprime stable P. I.-ring. Then the following are equivalent.

(a) $R$ is von Neumann regular.

(b) Each prime ideal of $R$ is maximal.

(c) Each prime factor ring of $R$ is von Neumann regular.

Proof. All that remains to be proven is that (c) implies (a). 
Consider the center $Z(R)$ of $R$. Since $R$ is semiprimitive, $Z(R) \neq 0$ by one of the implications of Formanek's work [12] on central polynomials. It follows from Theorem 2.3 that $R$ is $\pi$-regular and hence $Z(R)$ is $\pi$-regular by McCoy [21]. Hence $Z(R)$ is regular by Corollary 1.4 .

If $2 n$ is the degree of a polynomial of minimal degree which is satisfied by $R$, then $R$ satisfies all the identities of $n \times n$ matrices since $R$ is a subdirect product of simple Artinian rings - each of which has dimension equal to or less than $n^{2}$ over its center. Moreover, no homomorphic image of $R$ satisfies the identities of $(n-1) \times$ $(n-1)$ matrices. Whence $R$ is an Azumaya algebra by Proccesi's version of Artin's theorem [24]. Hence, the one-to-one correspondence given in [11, Corollary 3.7, p. 54] between the ideals of $R$ and the ideals of $Z(R)$ can be used to show that each ideal of $R$ is semiprime. Therefore $R$ is regular by Corollary 1.3.

THEOREM 2.6. If $R$ is a semiprime P. I.-ring and each prime factor ring of $R$ is von Neumann regular, then $R$ contains a von Neumann regular ideal which is essential.

Proof. By Brown-McCoy [9] $R$ contains a unique largest regular ideal which contains all regular ideals. Consequently, it will suffice to show that each nonzero ideal $A$ of $R$ contains a nonzero regular ideal.

First we show that $A$ inherits the hypotheses of $R$. Indeed, $A$ is semiprime P. I.-ring. Let $Q$ be a proper prime ideal of $A$. If we set $P=\{x \in R: A x \subseteq Q\}$, then it can be shown that $P$ is an ideal in $R$. Moreover, $P$ is prime, because $x, y \notin P$ implies $A x \nsubseteq Q$, $A y \nsubseteq Q$ implies $A x a y \nsubseteq Q$ or $x A y \nsubseteq P$. Furthermore, $Q=A \cap P$ and $A / Q \cong(A+P) / P=R / P$ since all prime ideals of $R$ are maximal.

If $n$ is the degree of a polynomial of minimal degree which is satisfied by $A$, then set $B=\bigcap\{Q: Q$ is a prime ideal of $A$ and $A / Q$ satisfies an identity of degree less than $n\}$. Note $B \neq 0$; otherwise $A$ satisfies a standard identity of degree less than $n$. More importantly, each prime factor ring of $B$ satisfies no identities of degree less than $n$.

Now $B$ inherits the hypotheses of $R$. As before $B$ has nontrivial center $Z(B)$ which is regular. Hence, there is a central idempotent $e$ in $Z(B)$. If $C$ is the ideal of $R$ generated by $e$, then $C$ is a semiprime P. I.-ring with unity in which each prime factor ring is regular. Moreover, $C$ is stable of degree $n$; otherwise, there is a nonzero prime factor ring of $C$ which satisfies an identity of degree less than $n$. However, each prime factor ring of $C$ is isomorphic to a prime factor ring of $B$ as above. This contradicts the 
fact that each prime factor ring of $B$ satisfies no identities of degree less than $n$. It now follows from Theorem 2.5 that $C$ is regular and the proof is complete.

COROLlaRy 2.7. If $R$ is a P. I.-ring in which every prime factor ring is von Neumann regular, then there exists a chain of ideals $\left\{I_{\alpha}\right\}$ with $I_{\alpha+1} / I_{\alpha}$ von Neumann regular or a nil ring and for each limit ordinal $\beta, I_{\beta}=\bigcup_{\alpha<\beta} I_{\alpha}$.

Proof. Let $I_{0}=B(R), I_{1} / I_{0}=$ maximal regular ideal of $R / I_{0}, I_{2}=$ $B\left(R / I_{1}\right)$, etc.

Armendariz-Fisher [5, Theorem 1] characterize von Neumann regular P. I.-rings as those in which two-sided ideals are idempotent. We obtain this as a corollary.

Corollary 2.8. Let $R$ be a P. I.-ring. Then $R$ is von Neumann regular if and only if $I^{2}=I$ for each two-sided ideal $I$ of $R$.

Proof. One implication is clear. For the other implication it is sufficient to show that each prime factor ring of $R$ is regular since then the maximal regular ideal of $R$ must coincide with $R$ by Theorem 2.6. If $S$ is a prime factor ring of $R$, then $S$ is fully idempotent and by Posner's theorem [22], $S$ has a simple Artinian classical two-sided quotient ring $Q$. The proof of Theorem 5(i) of Armendariz-Fisher [4] shows that each regular element in $S$ is invertible in $S$. Thus $S=Q$ and so $S$ is regular.

REMARK 4. This result is also a corollary of Theorem 1.1 since our proof shows that prime factor rings of $R$ are regular.

In Armendariz-Fisher [5] it is shown that a biregular P. I.-ring is von Neumann regular. The following example shows that the converse is false.

EXAMPLE 3. Let $F$ be a field which is embedded in $F_{2}$ via $F \rightarrow\left[\begin{array}{ll}f & 0 \\ 0 & 0\end{array}\right]$. Let $R$ consist of all sequences $\left\{a_{i}: a_{i} \in F_{2}\right\}$ for which (1) $a_{1} \in F$ and (2) there exists an $n$ depending on $\left\{a_{i}\right\}$ such that $a_{i}=$ $a_{i+n}$ for all $i$. Clearly $R$ is a regular P. I.-ring, yet $R$ is not biregular since the ideal generated by the constant sequence $\left[\begin{array}{ll}1 & 0 \\ 0 & 0\end{array}\right]$ is not generated by a central idempotent.

Nevertheless we do obtain the following.

THEOREM 2.9. If $R$ is a semiprime P. I.-ring in which each prime factor ring von Neumann regular, then $R$ contains a 
biregular ideal which is essential.

Proof. We will show that each nonzero ideal $A$ of $R$ contains a nonzero biregular ideal. This will guarantee that the largest biregular ideal of $R$ is essential. Theorem 2.3 yields that $R$ is a semisimple $\pi$-regular ring. Hence $R$ is a semisimple $I$-ring and each primitive factor ring of $R$ is of bounded index. By [14, Theorem 3 , p. 239] $A$ contains an ideal of the form $B_{n}$ where $B$ is a ring with unity and $B$ has no nilpotent elements. As in the proof of Theorem 2.6, $B_{n}$ is a semiprime P. I.-ring with regular prime factor rings. Since there is a one-to-one correspondence between the ideals of $B_{n}$ and $B$, each prime ideal of $B$ is maximal. Hence $B$ is regular by Corollary 1.4. Consequently, $B$ is biregular and by Andrunakievic [1] $B_{n}$ is biregular. This completes the proof.

REMARK 5. Theorem 2.6 can also be proven by using [14, Theorem 3, p. 239] and the techniques of this proof.

The authors wish to thank the referee for his helpful suggestions.

\section{REFERENCES}

1. V. A. Andrunakievic, Biregular rings, Math. Sbornik, 39 (81), (1956), 447-464.

2. V. A. Andrunakievic and Ju. M. Rjabuhin, Rings without nilpotent elements and completely simple ideals, Soviet Math. Dokl. 9 (1968), 565-568.

3. R. F. Arens and I. Kaplansky, Topological representation of algebras, Trans. Amer. Math. Soc., 63 (1948), 457-481.

4. E. P. Armendariz and Joe W. Fisher, Reduced P.I.-rings, (unpublished).

5. - Regular P.I.-rings, Proc. Amer. Math. Soc., 39 (1973), 247-251.

6. G. Azumapa, Strongly $\pi$-regular rings, J. Fac. Sci. Hokkaido Univ., 13 (1954), 34-39.

7. R. L. Blair, A note on f-regularity in rings, Proc. Amer. Math. Soc., 6 (1955), 511-513.

8. N. Bourbaki, Eléments de Mathématique, Fasc. XXVII Algebre commutative, Actualites Sci. Indust. No. 1290, Hermann Paris, 1961.

9. B. Brown and N. H. McCoy, The maximal regular ideal of a ring, Proc. Amer. Math. Soc., 1 (1950), 165-171.

10. R. C. Courter, Rings all of whose factor rings are semiprime, Canad. Math. Bull., 12 (1969), 417-426.

11. F. DeMeyer and E. Ingraham, Separable Algebras Over Commutative Rings, Lecture Notes in Mathematics, No. 181, Springer-Verlag, Berlin-New York, 1971.

12. E. Formanek, Central polynomials for matrix rings, J. Algebra, 23 (1972), 129132.

13. I. N. Herstein and Lance Small, Nil rings satisfying certain chain conditions: an addendum, Canad. J. Math., 18 (1966), 300-302.

14. N. Jacobson, Structure of Rings, Amer. Math. Soc. Coll. Publ. XXXVII, Providence, R. I., 1964.

15. S. K. Jain and Saroj Jain, Restricted regular rings, Math. Z., 121 (1971), 51-54. 16. I. Kaplansky, Rings with polynomial identity, Bull. Amer. Math. Soc., 54 (1948), $575-580$. 
17. I. Kaplansky, Topological representation of algebras, II, Trans. Amer. Math. Soc., 68 (1950), 62-75.

18. - Algebraic and Analytic Aspects of Operator Algebras, Amer. Math. Soc., Providence, R. I., 1970.

19. Lecture Notes, (unpublished).

20. J. Levitzki, A theorem on polynomial identities, Proc. Amer. Math. Soc., 1 (1950), $334-341$.

21. N. H. McCoy, Generalized regular rings, Bull. Amer. Math. Soc., 45 (1939), 175-178.

22. E. C. Posner, Prime rings satisfying a polynomial identity, Proc. Amer. Math. Soc., 11 (1960), 180-183.

23. C. Procesi, Sugli anelli non commutative zero dimensionali con identita polinomiale, Rediconte Circ. Mat. Palermo, 18 (1968), 5-12.

24. - On a theorem of M. Artin, J. Algebra 22 (1972) 309-315.

25. L. Rowen, Some results on the center of a rings with polynomial identity, Bull. Amer. Math. Soc., 79 (1973), 219-223.

26. Lance Small, Localization in P.I..rings, J. Algebra, 18 (1971, 269-270.

27. R. L. Snider, Rings with regular prime factor rings, (Unpublished).

28. S. A. Steinberg, Rings of quotients of rings without nilpotent elements, (to appear).

29. H. H. Storrer, Epimorphism en von kommutativen ringen, Comm. Math. Helv., 43 (1968), 378-401.

30. E. T. Wong, Regular rings and integral extension of a regular ring, Proc. Amer. Math. Soc., 33 (1972), 313-315.

31. O. Zariski and P. Samuel, Commutative Algebra, Vol. I, Van Nostrand, Princeton, N. J., 1958.

Received March 12, 1973 and in revised form October 2, 1973. The first author wishes to express his appreciation to Professor I. N. Herstein for inviting him to spend a stimulating summer, 1971, at the University of Chicago.

UNIVERSITY OF TEXAS

AND

NORTHWESTERN UNIVERSITY 


\section{PACIFIC JOURNAL OF MATHEMATICS}

\section{EDITORS}

RICHARD ARENS (Managing Editor)

University of California

Los Angeles, California 90024

R. A. Beaumont

University of Washington

Seattle, Washington 98105
J. DugundJI

Department of Mathematics University of Southern California Los Angeles, California 90007

D. Gilbarg AND J. Milgram Stanford University

Stanford, California 94305

\section{ASSOCIATE EDITORS}
E. F. BECKENBACH
B. H. NeUmanN
F. WOLF
K. YOSHIDA

\section{SUPPORTING INSTITUTIONS}

UNIVERSITY OF BRITISH COLUMBIA CALIFORNIA INSTITUTE OF TECHNOLOGY

UNIVERSITY OF CALIFORNIA

MONTANA STATE UNIVERSITY

UNIVERSITY OF NEVADA

NEW MEXICO STATE UNIVERSITY

OREGON STATE UNIVERSITY

UNIVERSITY OF OREGON

OSAKA UNIVERSITY
UNIVERSITY OF SOUTHERN CALIFOF

STANFORD UNIVERSITY

UNIVERSITY OF TOKYO

UNIVERSITY OF UTAH

WASHINGTON STATE UNIVERSITY UNIVERSITY OF WASHINGTON

AMERICAN MATHEMATICAL SOCIET NAVAL WEAPONS CENTER 


\section{Pacific Journal of Mathematics}

\section{Vol. 54, No. 1 \\ May, 1974}

Ralph K Amayo, Engel Lie rings with chain conditions ..................

Bernd Anger and Jörn Lembcke, Hahn-Banach type theorems for hypolinear

functionals on preordered topological vector spaces ..................

Gregory Frank Bachelis and Samuel Ebenstein, On $\Lambda(p)$ sets ................

Harvey Isaac Blau, Indecomposable modules for direct products of finite

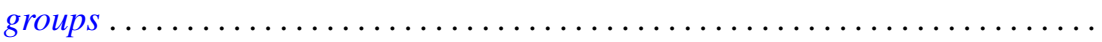

Larry Eugene Bobisud and James Calvert, Singular perturbation of a

time-dependent Cauchy problem in a Hilbert space ................

Walter D. Burgess and Robert Raphael, Abian's order relation and orthogonal

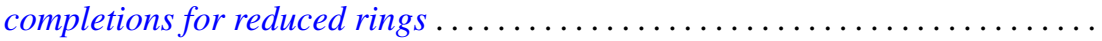

James Diederich, Representation of superharmonic functions mean continuous at

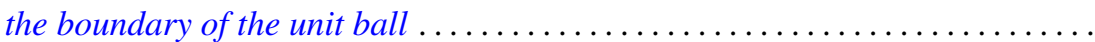

Aad Dijksma and Hendrik S. V. de Snoo, Self-adjoint extensions of symmetric

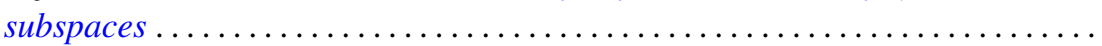

Gustave Adam Efroymson, A Nullstellensatz for Nash rings . . . . . . . . . . . . .

John D. Elwin and Donald R. Short, Branched immersions onto compact orientable surfaces . . . . . . . . . . . . . . . . . . . . . . . . .

John Douglas Faires, Comparison of the states of closed linear

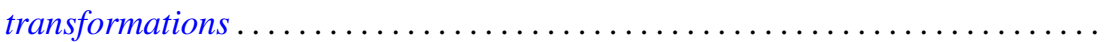

Joe Wayne Fisher and Robert L. Snider, On the von Neumann regularity of rings with regular prime factor rings .

Franklin Takashi Iha, A unified approach to boundary value problems on compact intervals

Palaniappan L. Kannappan and Che Tat $\mathrm{Ng}$, On functional equations connected with directed divergence, inaccuracy and generalized directed divergence

Samir A. Khabbaz and Elias Hanna Toubassi, The module structure of Ext $(F, T)$ over the endomorphism ring of $T$...

Garo K. Kiremidjian, On deformations of complex compact manifolds with boundary.

Dimitri Koutroufiotis, Mappings by parallel normals preserving principal

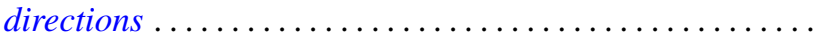

W. K. Nicholson, Semiperfect rings with abelian adjoint group

Norman R. Reilly, Extension of congruences and homomorphisms to translational hulls

Sadahiro Saeki, Symmetric maximal ideals in $M(G)$

Brian Kirkwood Schmidt, On the homotopy invariance of certain functors ...

H. J. Shyr and T. M. Viswanathan, On the radicals of lattice-ordered rings ...

Indranand Sinha, Certain representations of infinite group algebras ...

David Smallen, The group of self-equivalences of certain complexes ...

Kalathoor Varadarajan, On a certain problem of realization in homotopy

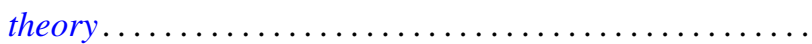

